\title{
Planejamento de uma montagem estrutural aeronaútica de jatos utilizando abordagens de programação de projetos com recursos restritos e múltiplos modos
}

\section{Planning of an aeronautical structural assembly of jets using project scheduling approach with constrained resources and multiple modes}

\author{
Bruno Jensen Virginio da Silva ${ }^{1}$ \\ Reinaldo Morabito
}

\begin{abstract}
Resumo: Neste estudo foi abordado um problema de planejamento de linha de montagem estrutural aeronáutica de jatos por meio de modelos de programação de projetos. Considera-se que a linha é dedicada (i.e., apenas um único tipo de produto é montado durante o horizonte de planejamento), o custo do estoque em processo não é diferenciado por segmento estrutural, nem por estação de trabalho, desconsidera-se o uso de horas extras para cumprir os prazos do plano e também o efeito da curva de aprendizagem. Foi avaliada a aplicação de modelos de programação de projetos com recursos restritos e com um ou múltiplos modos de execução das atividades. Também foi avaliado o impacto que o leadtime de montagem tem sobre a quantidade total e o nivelamento da mão de obra de montagem. Por exemplo, se para diferentes valores de leadtimes é possível diminuir a quantidade total de mão de obra necessária e quais seriam os efeitos dessa variação no estoque em processo. Para isso, diversos experimentos computacionais foram realizados utilizando-se casos realistas do problema de montagem estrutural de jatos em uma empresa aeronáutica.

Palavras-chave: Programação de produção; Indústria aeronáutica; Montagem estrutural de aviões; Nivelamento da mão de obra; Estoque em processo; Leadtime.
\end{abstract}

\begin{abstract}
This paper studies a production planning problem of an aircraft structural assembly line. The assembly line is dedicated (i.e., only one type of product is assembled during the planning time horizon), the work-in-process cost is not differentiated by structural segment neither by workstation, and the use of overtime to meet the plan deadline and the learning curve effect are disregarded. This study analyses the application of project scheduling models with limited resources and one or multiple execution modes for each activity. We also assessed the impact of the assembly leadtime on the total amount and level of workers, for instance, if for different values of leadtimes, it is possible to decrease the total amount of required workers and the effects of this change in the work-in-process. Several computational experiments were made using real problem instances of structural assembly of jets in an aeronautics company.
\end{abstract}

Keywords: Production scheduling; Aeronautic industry; Aircraft structural assembly; Manpower leveling; Work in process; Leadtime.

\section{Introdução}

As montagens estruturais de aviões são operações complexas que em geral utilizam recursos escassos e dispendiosos, como gabaritos de montagem e mão de obra especializada. Embora as montagens aeronáuticas possuam várias características da teoria de programação de projetos, são poucos os trabalhos reportados na literatura de engenharia de produção e pesquisa operacional que estudam problemas de planejamento e programação da produção de montagens estruturais de jatos. A otimização dos recursos produtivos utilizando técnicas de programação da produção e aplicativos especialistas oferece oportunidades importantes para realizar análises elaboradas, capazes de tornar o planejamento de montagens de aviões mais efetivo e contribuir para criar vantagens competitivas para as empresas aeronáuticas. Por exemplo, a simulação discreta é uma abordagem experimental utilizada quando o ambiente de decisão

\footnotetext{
${ }^{1}$ Departamento de Engenharia de Produção, Universidade Federal de São Carlos - UFSCar, São Carlos, SP, Brasil, e-mail: brunojen@gmail.com; morabito@ufscar.br
} 
é complexo (Banks, 1998), e alguns trabalhos são encontrados na literatura utilizando simulação com diversos enfoques em montagens aeronáuticas, tais como em Scott (1994) e Brown \& Caddick (2002).

$\mathrm{Na}$ gestão da produção aplicada à indústria aeroespacial, a principal fonte de trabalho são os estudos conduzidos pelo Lean Aerospace Initiative (LAI), realizados pelo Instituto de Tecnologia de Massachusetts (MIT) nos Estados Unidos, como o estudo da linha de montagem estrutural do Boeing 737 em Ferdowsi \& Haggerty (2002) e o estudo sobre a produção do míssil AMRAAM, descrito por Davidz \& Nightingale (2002). Wolters (2000) estudou a melhoria no sequenciamento da linha de montagem final do helicóptero UH-60 Blackhawk. Lennox (2006) realizou um estudo de implantação do sistema de produção puxada na Hamilton Sundstrand, fornecedora de sistemas da United Technologies (UTS). Em todos os casos, as técnicas usadas são derivadas das técnicas de manufatura enxuta, como gestão visual, mapeamento de fluxo de valor, logística JIT (Just in time), entre outras.

Esses trabalhos não reportam o uso de abordagens de otimização e programação matemática para auxiliar a tomada de decisão no planejamento das linhas de montagem aeronáuticas. Alguns trabalhos explorando essas abordagens tiveram como enfoque determinar a sequência de montagem durante a fase de projeto de estruturas aeronáuticas. Por exemplo, Zhang et al. (2008) propõem um algoritmo para selecionar sequencias ótimas de montagem em conjuntos aeronáuticos, respeitando vários critérios de projeto ao mesmo tempo. Silva (2010) e Silva et al (2011, 2014a, b) estudaram a programação da montagem de um subconjunto aeronáutico em gabaritos com restrições de adjacência utilizando modelos de programação de job shop com o objetivo de minimizar o makespan, e modelos de programação de projetos com recursos restritos com o objetivo de minimizar a quantidade total de mão de obra.

Outro estudo que aplica otimização matemática ao planejamento de montagens estruturais na indústria aeroespacial aparece em Heike et al. (2001). O estudo foi desenvolvido em uma linha de montagem final de aviões comerciais da Boeing. Os autores avaliaram quais os efeitos de trabalhar com tempos de ciclo constantes e variáveis, mão de obra dedicada ou flexível em uma linha mista, ou seja, em que são montados aviões diferentes conforme um plano de produção, com cargas de trabalho que variam entre os aviões, dependendo da complexidade da linha. O objetivo era minimizar o custo total, formado pelo custo da mão de obra alocada e das respectivas horas extras e do estoque em processo mantido na linha. Para isso, o trabalho propôs modelos de otimização inteiros mistos e não lineares, inspirados na literatura de modelos para linhas de montagem mistas. Segundo Heike et al. (2001), esses problemas estão presentes em outros contextos práticos, como linhas de montagem de carros, produtos eletrônicos e móveis.

O presente estudo aborda um problema de planejamento de linhas de montagem estruturais aeronáuticas de jatos por meio de modelos de programação de projetos. Algumas características do problema prático aqui estudado são diferentes de Heike et al. (2001). No presente estudo, a linha é dedicada, ou seja, apenas um único tipo de produto é montado durante o horizonte de planejamento, não é considerado o uso de horas extras para cumprir os prazos do plano e o custo do estoque em processo não é diferenciado por segmento estrutural, nem por estação de trabalho. Utilizando a rede de precedência das atividades do problema, foram avaliadas quais estratégias oferecem oportunidades de diminuir a quantidade total de mão de obra utilizada nas montagens, melhorando o nivelamento do seu uso, e também de diminuir o estoque em processo.

Um dos objetivos deste estudo foi aplicar e comparar a utilização de modelos de programação de projetos com recursos restritos e múltiplos modos de execução (Yamashita \& Morabito, 2007a, b, 2009; Brucker et al, 1999; Herroelen et al, 1998) com modelos de programação de projetos com recursos restritos, mas com um único modo de execução. Apesar de pertencerem a classes diferentes de problemas, o objetivo neste estudo é comparar a prática de programação atual (único modo) com o uso de um aplicativo especialista para programação de projetos com múltiplos modos de execução. Outro objetivo deste estudo foi avaliar qual o impacto que o leadtime de montagem tem sobre a quantidade total e o nivelamento da mão de obra de montagem. Por exemplo, avaliar se, para diferentes valores de leadtimes, é possível diminuir a quantidade total de mão de obra necessária e quais seriam os efeitos dessa variação no estoque em processo. Neste trabalho não é considerada a diminuição dos tempos de montagem com a evolução da produção em série, conhecida como curva de aprendizagem (Wright, 1936; Anzanello \& Fogliato, 2007).

Este trabalho está dividido em cinco seções. Na seção 2, o problema de planejamento de montagem estrutural é resumidamente descrito. Na seção 3 são apresentadas as modelagens matemáticas do problema, baseadas na programação de projetos com recursos restritos com um ou múltiplos modos de execução das atividades. Na seção 4 são apresentados e analisados os resultados dos experimentos computacionais aplicando os modelos para resolver casos realistas do problema. Finalmente, na seção 5 são apresentadas as considerações finais deste estudo e discutidas algumas perspectivas para pesquisa futura. 


\section{Descrição do problema}

A seguir, o problema de planejamento de montagem estrutural aeronáutica é descrito em mais detalhes e são apresentados os dados utilizados nos experimentos computacionais da seção 4. Essa descrição é motivada pelo problema encontrado na prática de uma empresa aeronáutica. Trata-se de uma linha de montagem de estruturas aeronáuticas de jatos, montadas em série, formadas por três segmentos estruturais. As montagens acontecem todas dentro de um determinado período, do mesmo modelo de avião, e estão localizadas no mesmo hangar. Após a etapa de montagem em estudo, as estruturas são enviadas para outros locais, onde a montagem final das estruturas e das aeronaves é concluída.

\subsection{Descrição das atividades da montagem aeronáutica}

Neste trabalho é adotada a nomenclatura de atividades para todas as atividades operacionais. Um conjunto de atividades relacionadas forma um projeto (Morton \& Pentico, 1993). As atividades são definidas como as atividades operacionais executadas na montagem estrutural pelos operadores de montagens estruturais, tipicamente chapeadores (montador mecânico) e seladores (responsáveis pela selagem de interfaces, uma forma de vedação das estruturas aeronáuticas). As atividades agrupadas formam a montagem de um único avião (ou do mesmo número de série, que é o nome sequencial dado a cada avião que é produzido; por exemplo, NS01 é o primeiro avião). O projeto é o conjunto das atividades que representam a montagem de todos os aviões que estão no plano tático de produção dentro do horizonte de tempo do planejamento em estudo.

Conforme ilustrado no exemplo da Tabela 1, a montagem de cada avião é formada por 24 atividades que utilizam 13 gabaritos de montagem diferentes ou vários carros de complementação específicos (dispositivos utilizados para apoiar as estruturas aeronáuticas enquanto são montadas), apresentados na coluna Recurso. O tipo de atividade define se é montagem ou selagem e, consequentemente, se utiliza montadores ou seladores. A análise da quantidade de seladores foi excluída do escopo deste estudo, por isso, todas as atividades de selagem não consomem recurso algum, nem homens-hora (HH) ou pessoas. Porém, as suas respectivas durações devem ser respeitadas para que o programa seja viável. Por simplicidade, neste exemplo as durações das operações de selagem e o tempo de cura não variam (ou seja, independem de temperatura, umidade, etc.) e não é considerado

Tabela 1. Duração, tipo e recursos de uma atividade.

\begin{tabular}{cccccc}
\hline Atividades & Duração & HH & Recurso & Tipo de atividade & Número de pessoas \\
\hline 1 & 4 & 4 & 1 & Montagem & 1 \\
2 & 2 & 2 & 2 & Montagem & 1 \\
3 & 2 & 2 & 3 & Montagem & 1 \\
4 & 2 & 2 & 4 & Montagem & 1 \\
5 & 4 & 16 & 5 & Montagem & 4 \\
6 & 4 & 12 & 6 & Montagem & 3 \\
7 & 3 & 0 & 0 & Selagem & 0 \\
8 & 1 & 3 & 7 & Montagem & 3 \\
9 & 6 & 12 & 8 & Montagem & 2 \\
10 & 7 & 35 & 9 & Montagem & 5 \\
11 & 8 & 32 & 10 & Montagem & 4 \\
12 & 4 & 0 & 0 & Selagem & 0 \\
13 & 6 & 6 & 11 & Montagem & 1 \\
14 & 4 & 8 & 12 & Montagem & 2 \\
15 & 4 & 8 & 13 & Montagem & 2 \\
16 & 4 & 4 & 14 & Montagem & 1 \\
17 & 6 & 12 & 15 & Montagem & 2 \\
18 & 2 & 4 & 16 & Montagem & 2 \\
19 & 6 & 24 & 17 & Montagem & 4 \\
20 & 5 & 15 & 18 & Montagem & 3 \\
21 & 3 & 0 & 0 & Selagem & 0 \\
22 & 8 & 16 & 19 & Montagem & 2 \\
23 & 6 & 12 & 20 & Montagem & 2 \\
24 & 5 & 0 & 0 & Selagem & 0 \\
\hline & & & &
\end{tabular}


o período da madrugada como tempo de cura; assim, o tempo de selagem e cura é conhecido e constante.

Os nomes e dados das montagens foram descaracterizados e distorcidos para proteger os interesses da empresa, porém manteve-se a proporcionalidade original dos dados. Além das atividades apresentadas na Tabela 1 , foram utilizadas duas atividades fantasmas ou fictícias: a atividade 0 , que precede todas as atividades, e a atividade 25 , que sucede todas as atividades, ambas com duração e quantidade de operadores nulos e sem utilizar nenhum tipo de recurso produtivo.

\subsection{Tempo de ciclo e leadtime}

O tempo de ciclo é o tempo que transcorre entre o término de dois aviões montados consecutivamente. Ele é determinado pela quantidade de tempo disponível para produção (por exemplo, dois turnos) e a quantidade de aviões a ser produzida no período, geralmente chamada de cadência de produção (por exemplo, aviões/mês). Para exemplificar, considere a cadência de produção de 10 aviões/mês e disponibilidade de 20 dias por mês para produzi-los. O tempo de ciclo é a relação entre o tempo disponível e a cadência de produção, calculado pela Expressão 1 .

$$
\text { Tempo de ciclo }=\frac{\text { tempo disponivel }}{\text { demanda }}=\frac{20 \text { dias }}{10 \text { aviões }}=\frac{2 \text { dias }}{\text { avião }}
$$

Note que o tempo de ciclo é um dado do problema, decorrente do plano de produção tático da empresa, e para os propósitos deste estudo, considera-se que ele não pode ser modificado.

O leadtime é o tempo transcorrido entre o início da primeira atividade e o término da última atividade de montagem de determinado número de série. As datas em que a última atividade de montagem de cada avião precisa ser concluída dependem do tempo de ciclo e do plano de produção da etapa seguinte, que ocorre em outros locais e são, por isso, dados do plano de produção e não podem ser alteradas. Já o momento de início da montagem de cada número de série pode ser decidido pelo planejamento da montagem em questão, por isso é analisado neste estudo.

A Figura 1 apresenta um exemplo de programação, considerando o leadtime de 5 u.t. (unidades de tempo) e tempo de ciclo de 2 u.t./avião. Observe que é possível identificar ambos graficamente, conforme indicado. É importante destacar que o gráfico é baseado no caso em que os leadtimes e tempos de ciclos são constantes e conhecidos previamente, ou seja, não considera incertezas nesses tempos.

Observando a Figura 1, é possível identificar o estoque em processo, ou seja, quantos aviões estão na linha de montagem ao mesmo tempo. Para isso, basta contar quantos aviões estão em montagem simultaneamente em um instante de tempo qualquer.
A Figura 2 apresenta a variação do estoque em processo do programa de produção exemplo da Figura 1. Observe que existe uma rampa de início, pois a linha de montagem inicia vazia, e depois uma rampa no final, pois a linha de montagem se esvazia ao final desse plano. Porém, quando em regime de equilíbrio, o estoque em processo (WIP - work in process) varia entre 2 e 3 aviões, regularmente.

É possível estimar o estoque em processo médio em uma montagem, conhecendo-se o leadtime médio e o tempo de ciclo, utilizando a conhecida relação:

$$
\text { WIP Médio }=\frac{\text { Leadtime médio }}{\text { Tempo de ciclo }}
$$

A Equação 2 é diretamente deduzível da fórmula de Little (Little, 1961). O estoque em processo médio (WIP médio) é equivalente ao número médio de usuários (aviões) em um sistema, o leadtime médio é o tempo de atravessamento do sistema (formado pela soma do tempo de fila e do tempo de processamento dos usuários) e o tempo de ciclo é o inverso da taxa média de chegadas de usuários no sistema (verificável na Expressão 1). Assim, o WIP médio do programa apresentado nas Figuras 1 e 2, quando em regime de equilíbrio, é calculado conforme a Equação 3:

$$
\text { WIP Médio }=\frac{5 \text { u.t. }}{2 \text { u.t. } / \text { avião }}=2,5 \text { aviões }
$$

Considerando que podemos ter diferentes planos de produção, cada um com um leadtime médio diferente para produzir um avião, definem-se os seguintes parâmetros do problema:

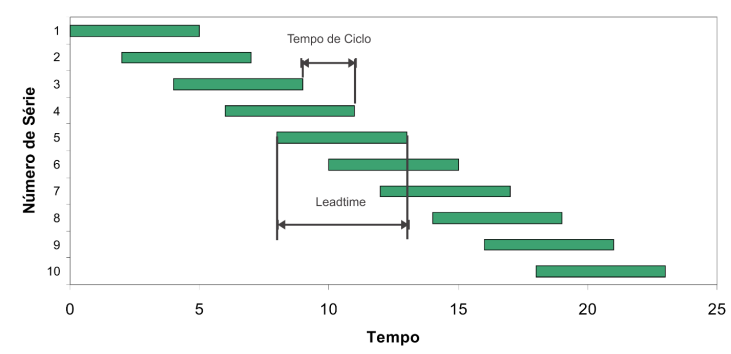

Figura 1. Gráfico de Gantt com o Tempo de Ciclo e Leadtime.

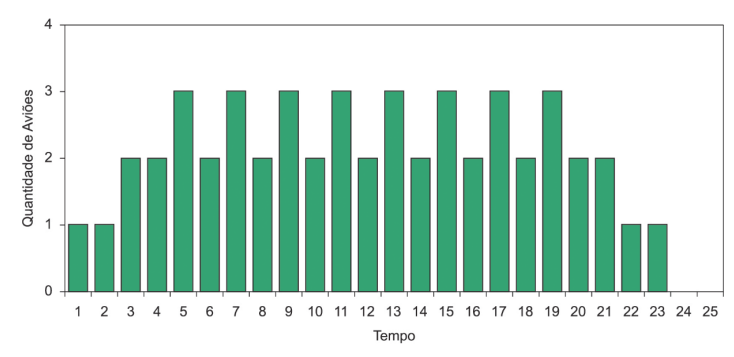

Figura 2. Quantidade de aviões em estoque em processo. 
a: custo de utilizar uma unidade de recurso de mão de obra.

$b$ : $\quad$ custo de oportunidade ao manter uma unidade do estoque em processo.

$M O_{i}$ : quantidade de mão de obra contratada para executar o plano de produção com leadtime médio $i$.

$W I P_{i}$ : quantidade média de estoque em processo no plano de produção com leadtime médio $i$.

$O$ custo total de montar os aviões no plano de produção com leadtime médio $i$ é dado pela soma dos custos de mão de obra e de estoque em processo com leadtime médio $i$.

$$
\text { CustoTotal }_{i}=a \cdot M O_{i}+b \cdot W I P_{i}
$$

O objetivo do problema é determinar o mínimo custo total, ou seja, $\min _{i}\left\{\right.$ Custo Total $\left._{i}\right\}$. Observe que o $W I P_{i}$ na Equação 4 é calculado pela Equação 3 usando o leadtime médio $i$ e a quantidade de mão de obra $M O_{i}$ é determinada pelo plano de produção. No texto que segue, por simplicidade, o leadtime médio é simplesmente chamado de leadtime.

\subsection{Rede de precedência}

No exemplo da Tabela 1, a montagem de um avião é formada por 4 grupos de atividades, em que 3 são montagens dos segmentos que podem ser realizadas em paralelo, e um quarto grupo que é precedido pelos três primeiros e une todos os segmentos. Por isso esse último grupo é chamado de junção de segmentos. Os 4 grupos formam a rede de precedência apresentada na Figura 3. Utilizando os dados da Tabela 1, é possível determinar o caminho crítico desta rede de precedência, formado pelo caminho que inicia na atividade 13 e termina na atividade 24 , destacado em vermelho e com duração 47 u.t. Isso significa que, mesmo com uma quantidade ilimitada de pessoas disponíveis, não é possível ter um leadtime menor do que 47 u.t. para essa rede de precedência utilizando uma programação com um único modo. Em azul estão destacadas as operações de selagem que não consomem recursos nesse estudo.

\subsection{Múltiplos modos de execução}

Neste estudo, múltiplos modos de execução foram levantados para cada atividade por meio de entrevistas com os montadores de uma empresa. A Tabela 2 apresenta os dados para o projeto considerando múltiplos modos - são utilizados quatro modos de execução. Para cada modo de execução, é relacionada a quantidade de mão de obra empregada e a respectiva duração da atividade requerida neste modo, não sendo necessário manter uma relação linear ou qualquer outra. Nesse estudo, por simplicidade foi considerado que a quantidade total de homens-hora utilizada não varia com a mudança do modo de execução, exceto quando for necessário algum arredondamento. Por exemplo, a atividade 11 precisa de 32 homens-hora e é realizada em 8 u.t. com quatro pessoas, 16 u.t. com duas pessoas e, seguindo essa lógica, seria 6,4 u.t. com cinco pessoas, mas esse valor foi arredondado para 7 u.t. em decorrência de o modelo matemático utilizado considerar apenas durações inteiras, devido ao tempo ser discretizado em períodos constantes. Caso fosse utilizada uma formulação em tempo contínuo para o problema de programação de projetos com múltiplos modos de execução, esse arredondamento não seria necessário.

Utilizando os dados da Tabela 2 e a rede de precedência da Figura 3, é possível recalcular o caminho crítico e a sua duração, considerando que cada atividade é executada no modo com o menor tempo entre todos os modos de execução, ou seja, desconsiderando limitações do número total de pessoas.

A Figura 4 apresenta a duração de cada trecho da rede de precedência, supondo o modo mais rápido de executar cada atividade. Nesta figura observam-se vários caminhos na rede de precedência. Os caminhos com maior duração são (9-24) e (13-24), com duração total de 30 u.t., desconsiderando limitações do número de pessoas. Portanto, a rede possui dois caminhos críticos paralelos, destacados em vermelho, e a duração desses caminhos críticos é de 30 u.t.

\section{Modelagem matemática}

Neste estudo são aplicados dois modelos de otimização para abordar o problema. O primeiro é um modelo de programação de projetos com recursos restritos (RCPSP - resource constrained project scheduling problem) com um único modo de execução. O segundo é um modelo de programação de projetos com recursos restritos e múltiplos modos de execução (MMRCPSP - multi-mode resource constrained project scheduling problem). As formulações apresentadas a seguir e utilizadas nos testes computacionais são baseadas nos modelos da literatura que podem ser encontradas, por exemplo, em Morton \& Pentico (1993), Brucker et al. (1999) e Pinedo (2008). As unidades de tempo são discretas e os modelos possuem variáveis lineares inteiras, portanto, são modelos de programação linear inteira.

\subsection{Modelo com único modo de execução}

Os modelos de literatura definem dois tipos de recursos. O primeiro tipo refere-se a recursos em que a quantidade disponível é fixa e por isso são parâmetros do modelo. $\mathrm{O}$ segundo tipo refere-se a recursos variáveis, em que a quantidade a ser alocada é uma variável de decisão. Neste estudo, a linha de montagem tem dois tipos de recursos principais que são relevantes para o contexto: os gabaritos de montagem e os montadores. A quantidade de cada 
gabarito $k$ é fixa. Por outro lado, a mão de obra é um recurso variável a ser otimizado e é identificado pelo índice $w$. No caso de existir apenas um tipo de mão de obra, os chapeadores, as variáveis de decisão do modelo não precisam ter o índice $w$ da formulação clássica. Os parâmetros do modelo são:

Tabela 2. Duração das atividades com múltiplos modos de execução.

\begin{tabular}{|c|c|c|c|c|c|c|c|c|c|}
\hline \multirow[t]{2}{*}{ Atividades } & \multirow[t]{2}{*}{ HH } & $\begin{array}{c}\text { Duração } \\
(\text { u.t) }\end{array}$ & $\begin{array}{c}\text { Número } \\
\text { de } \\
\text { pessoas }\end{array}$ & $\begin{array}{c}\text { Duração } \\
\text { (u.t) }\end{array}$ & $\begin{array}{c}\text { Número } \\
\text { de } \\
\text { pessoas }\end{array}$ & $\begin{array}{c}\text { Duração } \\
\text { (u.t) }\end{array}$ & $\begin{array}{c}\text { Número } \\
\text { de } \\
\text { pessoas }\end{array}$ & $\begin{array}{c}\text { Duração } \\
\text { (u.t) }\end{array}$ & $\begin{array}{c}\text { Número } \\
\text { de } \\
\text { pessoas }\end{array}$ \\
\hline & & \multicolumn{2}{|c|}{ Modo 1} & \multicolumn{2}{|c|}{ Modo 2} & \multicolumn{2}{|c|}{ Modo 3} & \multicolumn{2}{|c|}{ Modo 4} \\
\hline 1 & 4 & 4 & 1 & 2 & 2 & 1 & 4 & - & - \\
\hline 2 & 2 & 2 & 1 & 1 & 2 & - & - & - & - \\
\hline 3 & 2 & 2 & 1 & 1 & 2 & - & - & - & - \\
\hline 4 & 2 & 2 & 1 & 1 & 2 & - & - & - & - \\
\hline 5 & 16 & 4 & 4 & 16 & 1 & 8 & 2 & 6 & 3 \\
\hline 6 & 12 & 4 & 3 & 6 & 2 & 12 & 1 & 3 & 4 \\
\hline 7 & 0 & 3 & 0 & - & - & - & - & - & - \\
\hline 8 & 3 & 1 & 3 & 3 & 1 & 2 & 2 & - & - \\
\hline 9 & 12 & 6 & 2 & 12 & 1 & 4 & 3 & 3 & 4 \\
\hline 10 & 35 & 7 & 5 & 18 & 2 & 12 & 3 & 5 & 7 \\
\hline 11 & 32 & 8 & 4 & 16 & 2 & 7 & 5 & 6 & 6 \\
\hline 12 & 0 & 4 & 0 & - & - & - & - & - & - \\
\hline 13 & 6 & 6 & 1 & 3 & 2 & 2 & 3 & - & - \\
\hline 14 & 8 & 4 & 2 & 8 & 1 & 3 & 3 & 2 & 4 \\
\hline 15 & 8 & 4 & 2 & 8 & 1 & 3 & 3 & 2 & 4 \\
\hline 16 & 4 & 4 & 1 & 2 & 2 & 1 & 4 & - & - \\
\hline 17 & 12 & 6 & 2 & 12 & 1 & 4 & 3 & 3 & 4 \\
\hline 18 & 4 & 2 & 2 & 4 & 1 & 2 & 3 & 1 & 4 \\
\hline 19 & 24 & 6 & 4 & 12 & 2 & 8 & 3 & 5 & 5 \\
\hline 20 & 15 & 5 & 3 & 15 & 1 & 8 & 2 & 4 & 4 \\
\hline 21 & 0 & 3 & 0 & - & - & - & - & - & - \\
\hline 22 & 16 & 8 & 2 & 16 & 1 & 6 & 3 & 4 & 4 \\
\hline 23 & 12 & 6 & 2 & 12 & 1 & 4 & 3 & 3 & 4 \\
\hline 24 & 0 & 5 & 0 & - & - & - & - & - & - \\
\hline
\end{tabular}

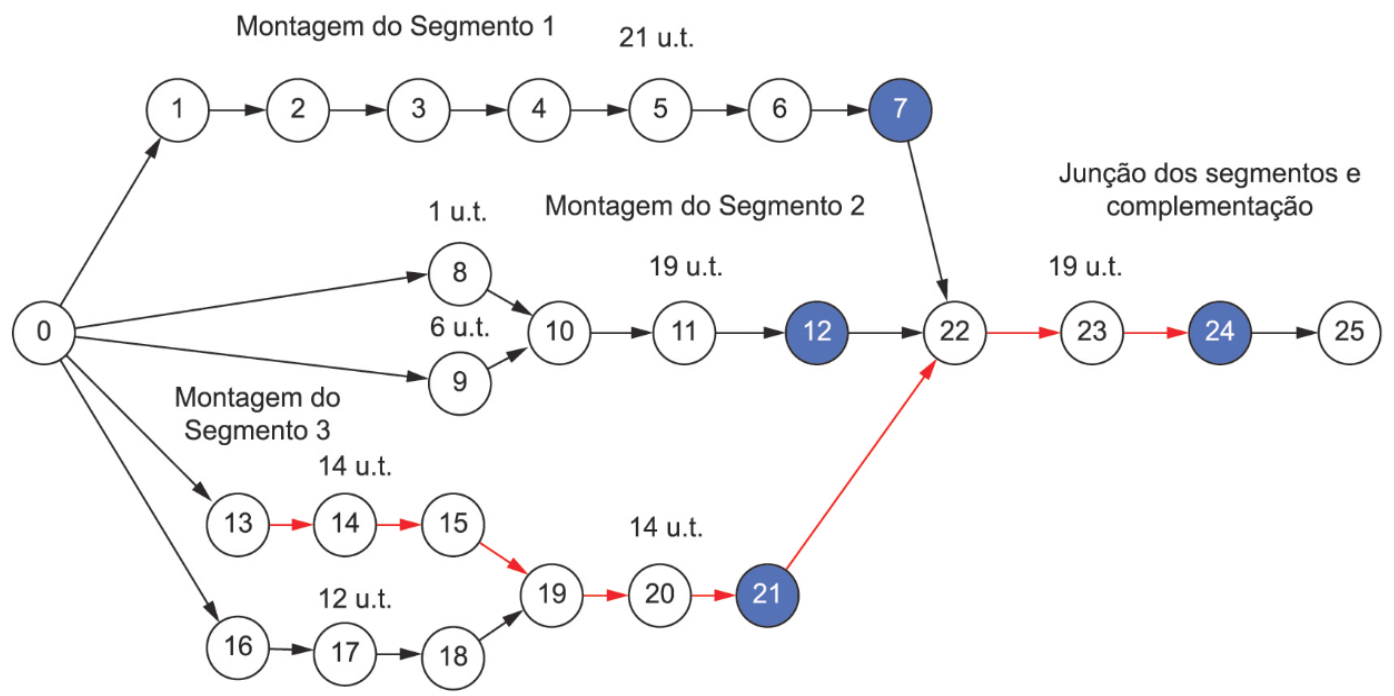

Figura 3. Rede de precedência da montagem da estrutural de um avião. 


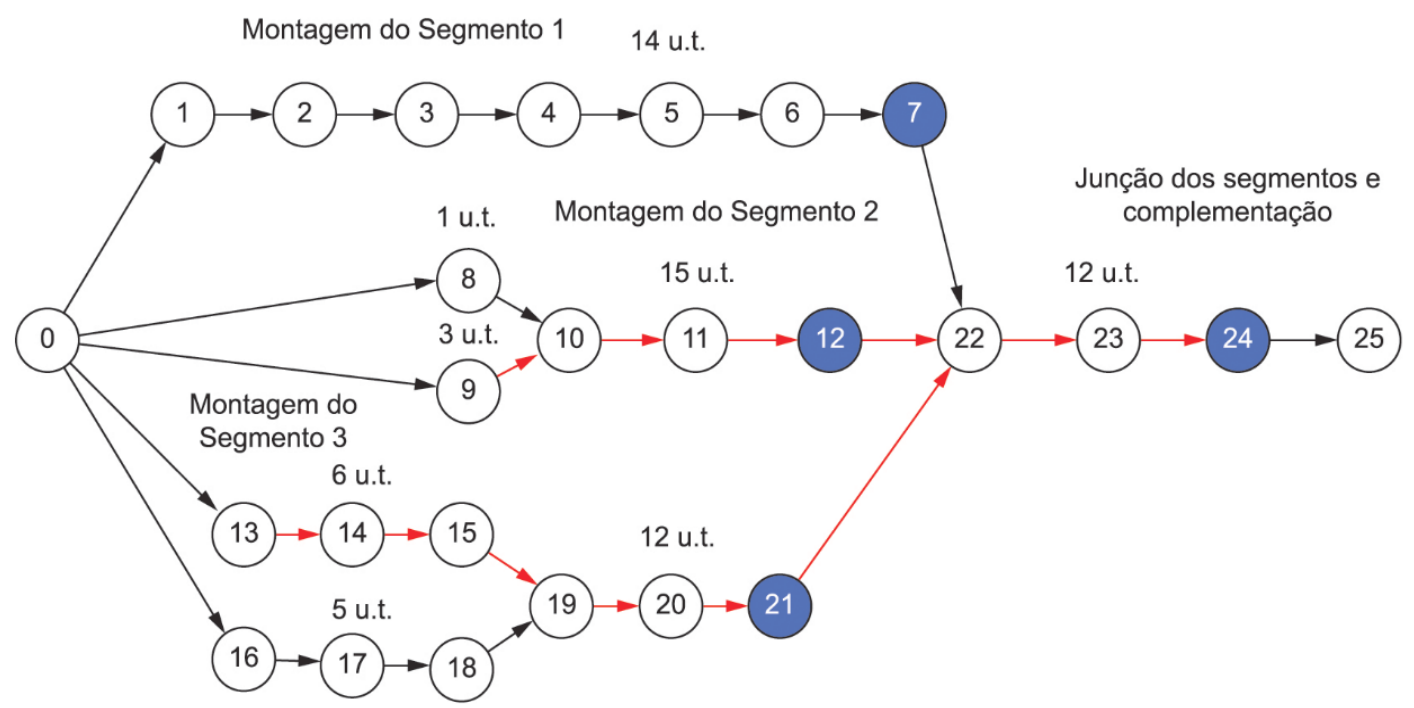

Figura 4. Rede de precedência com as atividades executadas no modo mais rápido.

$j \quad$ atividade, $j=1, \ldots, J$

$t \quad$ tempo, $t=1, \ldots, T$

$k \quad$ tipo de recursos (gabaritos), $k=1, \ldots, K$

$w \quad$ tipo de mão de obra, $w=1, \ldots, W$

$r_{j} \quad$ tempo em que a atividade $j$ está disponível, sendo que $1 \leq r_{j} \leq T$ e inteiro

$d_{j}$ tempo de entrega da atividade $j$, sendo que $1 \leq d_{j} \leq T$ e inteiro

$r e c_{j k}$ quantidade do recurso $k$ necessário (gabaritos) para executar a atividade $j$

$c_{k} \quad$ quantidade disponível do recurso (gabarito) $k$

$p_{j} \quad$ duração da atividade $j$

$m o_{j w}$ quantidade de mão de obra do tipo $w$ utilizada para executar a atividade $j$

$v_{w} \quad$ custo unitário da mão de obra do tipo $w$

Além disso, define-se o seguinte conjunto de pares de atividade $(h, j)$, sendo $h \neq j$.

$H=\{(h, j) \mid$ a atividade $h$ precede a atividade $j\}$.

$G=\{j \mid$ a atividade $j$ é executada no gabarito $\}$

As variáveis de decisão são:

$x_{j t}=\left\{\begin{array}{l}1 \text { se a atividade } j \text { termina no tempo } t \\ 0 \text { caso contrário }\end{array}\right.$

$a_{w} \quad$ quantidade de mão de obra do tipo $w$ a ser disponibilizada

Uma formulação para o RCPSP com um modo de execução é dada por:

$$
\begin{gathered}
\operatorname{Min} \sum_{w=1}^{W} v_{w} a_{w} \\
\sum_{t=r_{j}}^{d_{j}} x_{j t}=1 \text { para } \forall j
\end{gathered}
$$

$$
\begin{gathered}
\sum_{t=r_{h}}^{d_{h}} t . x_{h t} \leq \sum_{t=r_{j}}^{d_{j}}\left(t-p_{j}\right) . x_{j t} \text { para } \forall(h, j) \in H \\
\sum_{j \in G} \sum_{b=t}^{t+p_{j}-1} r e c_{j k} x_{j b} \leq c_{k} \text { para } \forall(k, t) \\
\sum_{j=1}^{J} \sum_{b=t}^{t+p_{j}-1} m o_{j w} x_{j b} \leq a_{w} \text { para } \forall(w, t) \\
t=1, \ldots, r_{j}+p_{j}-2 \quad \operatorname{para} \forall j \\
x_{j t}=0 \quad t=d_{j}+1, \ldots, T \\
x_{j t} \in\{0,1\} ; a_{w} \in Z^{+}, j=1, \ldots, J ; t=1, \ldots, T ; w=1, \ldots, W ; k=1, \ldots, K
\end{gathered}
$$

A Função objetivo 5 minimiza o custo total do projeto, representado pela quantidade de mão de obra utilizada no plano, multiplicada pelo custo unitário de utilizá-la. As Restrições 6 obrigam que todas as atividades do plano sejam programadas. As Restrições 7 garantem a relação de precedência entre as atividades. As Restrições 8 e 9 garantem que a atividade só seja programada se houver recursos $c_{k}$ e $a_{w}$ disponíveis durante sua execução. As Restrições 10 eliminam variáveis desnecessárias (alternativamente, essas variáveis poderiam ter sido desconsideradas na definição do modelo, eliminando a necessidade destas restrições). As Restrições 11 referem-se ao domínio das variáveis.

\subsection{Modelo com múltiplos modos de execução}

A linha de produção estudada também pode ser abordada como um problema de programação de projetos com múltiplos modos de execução (MMRCPSP). Neste caso, cada atividade da rede de precedência pode ser realizada de vários modos, cada 
modo utiliza diferentes quantidades de mão de obra e, consequentemente, têm diferentes durações. Assim, a utilização de um MMRCPSP é uma alternativa interessante para comparação do desempenho do RCPSP. Considere os parâmetros anteriores e os seguintes parâmetros adicionais:

$r e c_{j i k}$ quantidade de recursos necessários para executar a atividade $j$ no modo de execução $i$

$p_{j i} \quad$ duração da atividade $j$ no modo $i$

$m o_{j i w}$ quantidade de mão de obra do tipo $w$ utilizada para executar a atividade $j$ no gabarito no modo $i$

As variáveis de decisão são:

$x_{j t i}=\left\{\begin{array}{l}1 \text { se a atividade } j \text { termina no tempo } t \\ \text { no modo } i 0 \text { caso contrário }\end{array}\right.$

$a_{w}$ quantidade de mão de obra do tipo $w$ a ser disponibilizada

Uma formulação para o MMRCPSP com múltiplos modos de execução é dada por:

$$
\begin{gathered}
\operatorname{Min} \sum_{w=1}^{W} v_{w} a_{w} \\
\sum_{i=1}^{I} \sum_{t=r_{j}}^{i_{w j}} x_{j i t}=1 \text { para } \forall j \\
\sum_{i=1}^{I} \sum_{t=r_{h}}^{d_{h}} t . x_{h t i} \leq \sum_{i=1}^{I} \sum_{t=r_{j}}^{d_{j}}\left(t-p_{j i}\right) x_{j i t} \text { para } \forall(h, j) \in H \\
\sum_{i=1}^{I} \sum_{j \in G} \sum_{b=t}^{t+p_{j}-1} r e c_{j k i} x_{j b i} \leq c_{k} \quad \operatorname{para} \forall(k, t) \\
\sum_{j \in J \backslash G} \sum_{b=t}^{t+p_{j}-1} m o b_{j w i} x_{j b i} \leq a_{w} \quad \forall(i, w, t) \\
t=1, \ldots, r_{j}+p_{j i}-2 \quad \forall(j, i) \\
t=d_{j}+1, \ldots, T \\
x_{j i t}=0 \quad\{0,1\} ; a_{w} \in Z^{+}, j=1, \ldots, J ; t=1, \ldots, T ; \\
w=1, \ldots, W ; k=1, \ldots, K, i=1, \ldots, I
\end{gathered}
$$

Observe que o modelo com múltiplos modos é semelhante ao modelo com único modo de execução, acrescentando o índice $i$ que identifica o modo de execução nas variáveis e modificando algumas restrições, acrescentando alguns somatórios. A Função objetivo 12 minimiza o custo total do projeto. As Restrições 13 garantem que todas as atividades são alocadas em apenas um único modo no plano. As Restrições 14 garantem a relação de precedência entre as atividades. As Restrições 15 e 16 garantem que as atividades só são alocadas se existirem recursos $c_{k} \mathrm{e}$ $a_{k}$ disponíveis durante a sua execução. As Restrições
17 eliminam variáveis desnecessárias. As Restrições 18 são referentes ao domínio das variáveis.

\section{Experimentos computacionais}

Foram realizados 238 experimentos computacionais (119 com o modelo (5)-(11) com único modo e $119 \mathrm{com}$ o modelo (12)-(18) com múltiplos modos), divididos em dois grupos, utilizando os dados da Tabela 1 e Tabela 2 e a rede de precedência da Figura 3, e dados de leadtime apresentados a seguir.

O primeiro grupo de experimentos tem o objetivo de avaliar o impacto do leadtime nos recursos e no custo total. O segundo grupo tem o objetivo de comparar a utilização da abordagem de único modo com a abordagem de múltiplos modos. Os experimentos foram realizados em um computador com processador Intel i7 2600K 3,4 GHz com 16 GB de RAM e 1 hora de processamento para cada problema. Todos os experimentos foram feitos no sistema operacional Windows 7, utilizando o GAMS 23.0.2 e o CPLEX 11.2.1, com a opção de 4 núcleos de processamento ativada e demais parâmetros nos seus valores default.

Em todos os experimentos não foi permitido modificar as quantidades de gabaritos na linha de montagem, por isso, vários problemas com os leadtimes e tempos de ciclo menores foram provados infactíveis pelo CPLEX. Em outros problemas, o CPLEX não encontrou solução inteira dentro do tempo limite predeterminado de 1 hora. Para a maior parte dos experimentos, uma solução ótima ou uma boa solução factível foi encontrada.

\subsection{Relação entre o leadtime, tempo de ciclo e quantidade de mão de obra}

Para essa análise, resolveram-se 119 problemas, variando o leadtime e o tempo de ciclo para os tempos de atividade do NS01 com o modelo de único modo, ou seja, o modelo (5)-(11). O objetivo do experimento era encontrar qual a quantidade mínima necessária de operadores para um dado leadtime e um dado tempo de ciclo da rede de precedência da montagem.

Os tempos de ciclo utilizados foram: 2, 3, 4, 5, $6,7,8,9,10,11,12,13,14,15,19,26$ e 40 . Os leadtimes utilizados foram 31, 35, 40, 45, 50, 55 e 60 . A Figura 5 apresenta a curva de mão de obra para o tempo de ciclo 10 e diferentes leadtimes do avião NS01. Observe que conforme o leadtime aumenta, para um mesmo tempo de ciclo, a quantidade de mão de obra necessária diminui. Nesse caso, comparando o leadtime 31 com o leadtime 60 , houve diminuição de 24 para 15 montadores, ou seja, cerca de $40 \%$ de redução. Esse efeito era esperado, pois, com um leadtime maior, é possível encontrar programas de produção que conseguem nivelar melhor a mão de obra e, por isso, a quantidade total de operadores necessários é menor. A curva que melhor se ajustou aos dados pelo 
critério do quadrado do coeficiente de determinação $R^{2}$ foi a curva geométrica apresentada na Figura 5 (a variável $y$ é a quantidade de montadores e a $x$ é o leadtime de montagem de cada avião). Observe que a variação segue um padrão de decaimento, alcançando um nível de montadores em que o aumento no leadtime impacta pouco na diminuição da quantidade de mão de obra alocada ao projeto.

A Figura 6 e a Figura 7 apresentam os nivelamentos da programação de produção para os leadtimes 31 e 35, respectivamente, apresentados na Figura 5. Observe que o nivelamento do programa de produção da Figura 7 é melhor que o da Figura 6, com uma menor quantidade de picos e vales. Observe também que a quantidade máxima de montadores é menor na Figura 7 (19 montadores) do que na Figura 6 (24 montadores), com uma redução de cerca de $20 \%$ na quantidade de montadores.

A taxa de ocupação da mão de obra aumenta com o aumento do leadtime, como esperado, em decorrência do melhor nivelamento. A Figura 8 apresenta a variação da taxa de ocupação para o NS01 com tempo de ciclo 10. Observe que para o leadtime

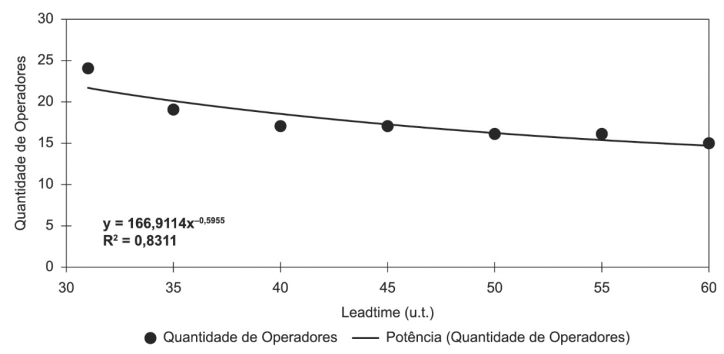

Figura 5. Variação da quantidade de operadores em função do leadtime com o tempo de ciclo de 10 .

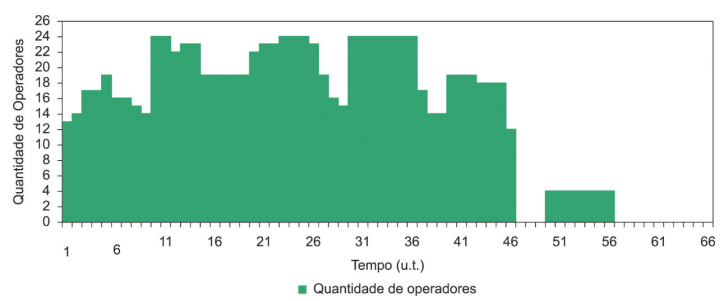

Figura 6. Nivelamento da mão de obra para o leadtime 31 e tempo de ciclo 10 .

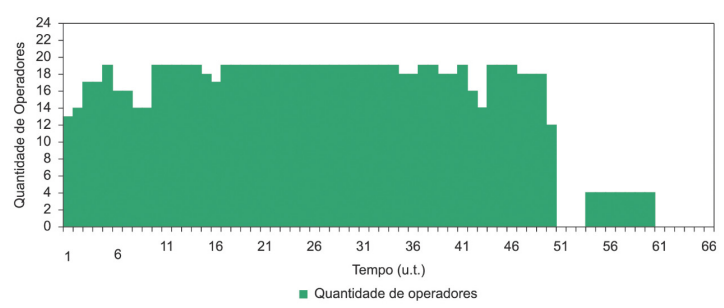

Figura 7. Nivelamento da mão de obra para o leadtime 35 e tempo de ciclo 10 .
60 u.t., a taxa de ocupação é de $85 \%$, o que significa que se a rede de precedência permitir, o aumento do leadtime poderia diminuir ainda mais a quantidade de mão de obra utilizada, até chegar hipoteticamente muito próximo à taxa de $100 \%$. Na Figura 8 também é apresentada a curva logarítmica que descreve a variação da taxa de ocupação (variável $y$ ) em função do leadtime (variável $x$ ).

Um resultado bem conhecido da teoria de filas é que, em sistemas de filas com processos de chegada e processamento probabilísticos (que não é o caso deste estudo), quanto maior a taxa de ocupação dos recursos, maior o estoque em processo, tendendo assintoticamente a infinito para valores próximos de $100 \%$ (Arenales et al., 2007). Nesse estudo, os dados são considerados todos constantes, o que é uma simplificação da realidade. Por isso, a taxa de ocupação de $100 \%$ é uma idealização, ou seja, um limitante inferior teórico para a quantidade de operadores que só pode ser atingido sem gerar estoques infinitos sob a suposição de parâmetros determinísticos no sistema de produção.

A Figura 9 apresenta a variação da quantidade de mão de obra em função do leadtime e do tempo de ciclo. Os pontos com quantidade de mão de obra nula na figura são aqueles para os quais não foram encontradas soluções factíveis para o modelo dentro do tempo computacional limite, ou foram provados infactíveis pelo CPLEX. Observe no gráfico que há uma diminuição da quantidade de operadores com o aumento do leadtime em todos os tempos de ciclo do experimento. Observe também que existe uma queda

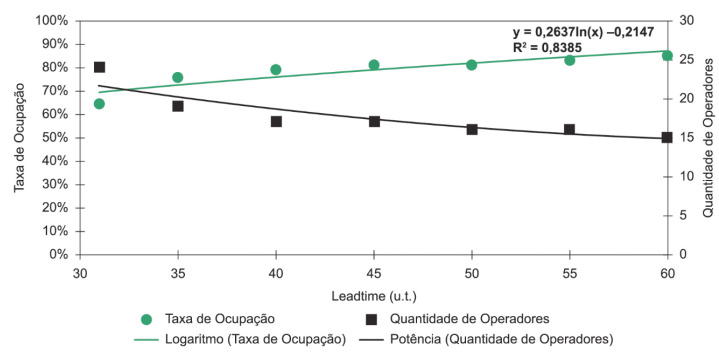

Figura 8. Variação da taxa de ocupação e da quantidade de mão de obra em função do leadtime para o tempo de ciclo de 10 u.t. e NS01.

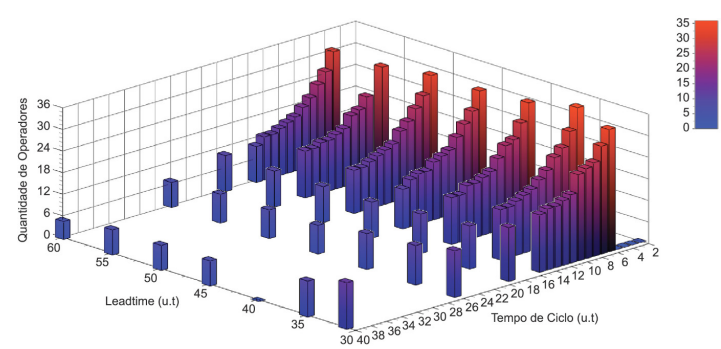

Figura 9. Variação da quantidade de mão de obra em função do leadtime e do tempo de ciclo do NS01. 
acentuada da quantidade de mão de obra conforme o tempo de ciclo aumenta, o que é explicável pela diminuição proporcional da quantidade de aviões produzidos no período. A maior parte dos experimentos computacionais envolveu tempos de execução menores que 1000 segundos e alguns poucos atingiram o tempo limite de 3600 segundos.

A Figura 10 apresenta um resumo da variação proporcional da quantidade de mão de obra em função da variação proporcional do leadtime. Observe que aumentar o leadtime de 31 para 60 u.t., ou seja, aumentá-lo em $94 \%$ causa reduções diferentes para diferentes tempos de ciclo. Por exemplo, para o tempo de ciclo 5 u.t., um aumento de $71 \%$ no leadtime (de 35 para 60 u.t.) gerou uma redução na quantidade de montadores necessários para realizarem todas as montagens de 36 para 30 montadores, ou seja, redução de $17 \%$. Para o tempo de ciclo 40, um aumento de $94 \%$ do leadtime gerou uma redução de 13 para 5 montadores, ou seja, $62 \%$ da mão de obra. Para os tempos de ciclo 2, 3 e 4 u.t. não foram encontradas soluções factíveis, mesmo após aumentar o leadtime.

\section{2 Único modo versus múltiplos modos de execução}

Nesse segundo conjunto de experimentos, foram gerados vários casos com diferentes leadtimes e tempos de ciclo considerando quatro modos de execução, para o caso com múltiplos modos, e um único modo de execução, conforme utilizado no planejamento da empresa. Foi possível encontrar a solução ótima

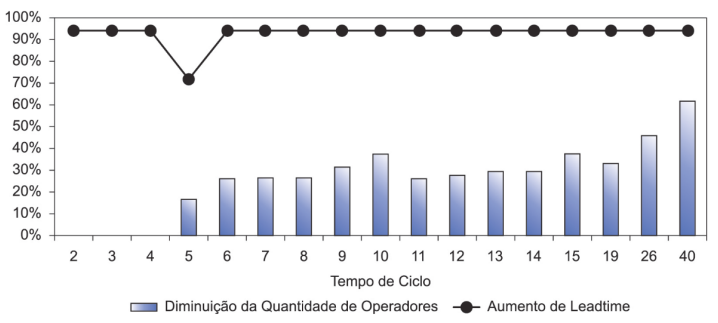

Figura 10. Variação proporcional da mão de obra em função da variação proporcional do leadtime.

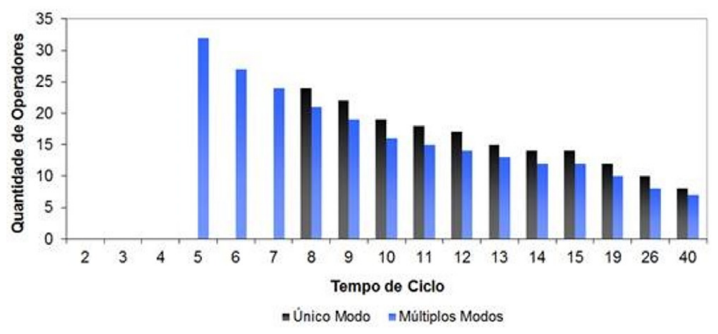

Figura 11. Comparação entre as soluções dos casos com único modo com os casos múltiplos modos para o leadtime 40. do programa de produção para ambos os casos - os resultados para o leadtime 40 para diferentes tempos de ciclo são apresentados na Figura 11. Como o problema com único modo é um caso particular do problema com múltiplos modos, a solução ótima do problema com único modo não pode ser melhor do que a solução do problema com múltiplos modos. Os resultados mostram que para os tempos de ciclo 5, 6 e 7 u.t., o problema com único modo é infactível, enquanto o problema com múltiplos modos tem solução. Para os tempos de ciclo iguais ou maiores do que 8 u.t., as soluções ótimas do problema com múltiplos modos são melhores do que as soluções ótimas do problema com único modo.

Considerando todos os experimentos com leadtime variando de 31 a 60 e tempo de ciclo variando de 2 a 40, em 90\% dos casos o uso de único modo de execução não resulta em soluções factíveis. Os problemas com múltiplos modos encontraram soluções para $48 \%$ dos casos em que o problema com único modo não encontrou, e em $10 \%$ dos experimentos, o uso de múltiplos modos melhorou a solução encontrada. Nos casos estudados, a utilização de múltiplos modos de execução diminuiu a quantidade total de montadores em média $18 \%$ em relação ao caso com único modo.

A Figura 12 apresenta a quantidade de mão de obra necessária para montar os aviões em cada leadtime e tempo de ciclo. Ao compará-la com a Figura 9, é visível a redução da quantidade de montadores necessários para os diversos tempos de ciclo e leadtimes. Observe também que a redução é maior para os tempos de ciclo menores e leadtimes maiores. Na maior parte dos experimentos computacionais, o tempo de execução foi inferior a 1000 segundos, com alguns alcançando o tempo máximo de 6000 segundos.

\subsection{Trade-off entre estoque e capacidade}

Com os resultados apresentados na seção 4.1, é possível calcular o custo total de alocação de mão de obra em cada tempo de ciclo e leadtime. Pode-se também calcular o estoque em processo médio para cada tempo de ciclo e leadtime utilizando-se a Expressão 1. Conhecendo o estoque em processo

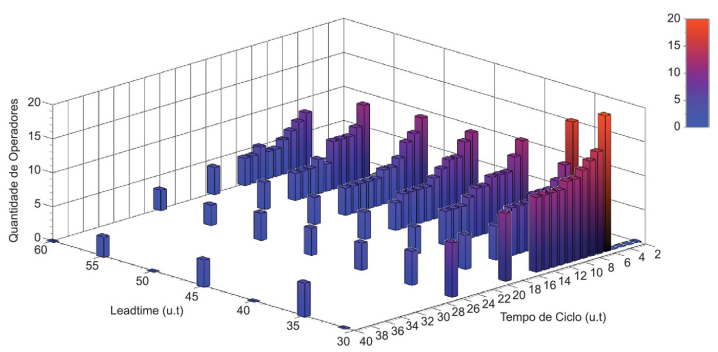

Figura 12. Variação da quantidade de mão de obra em função do leadtime e do tempo de ciclo do NS01 para o modelo com múltiplos modos de execução. 
médio e a quantidade de operadores, pode-se calcular o custo total utilizando-se a Expressão 3. A Figura 13 apresenta os resultados dessa análise para o tempo de ciclo 10 u.t. e NS01. Observe que o custo do estoque em processo cresce linearmente com o leadtime, enquanto o custo de mão de obra decai não linearmente com o aumento do leadtime, até se estabilizar em um patamar mínimo. Dessa maneira fica caracterizada a curva de trade-off entre o custo de capacidade e o custo de estoque em processo para o tempo de ciclo 10 u.t. e NS01. No caso apresentado, o ponto de custo total mínimo ocorre com o leadtime 40 para uma taxa de juros de $7 \%$ ao ano para custear o estoque em processo. Nesse caso, o custo do estoque em processo é muito maior do que o custo de capacidade ociosa; dessa forma, o leadtime com o custo total mínimo está relativamente próximo ao tempo crítico do projeto. O custo de cada avião no estoque em processo é de cerca de $\$ 3,2$ milhões, ou seja, a $7 \%$ ao ano o custo de oportunidade é de $\$ 225$ mil por avião, e cada montador alocado a essa montagem custa cerca de $\$ 56$ mil.

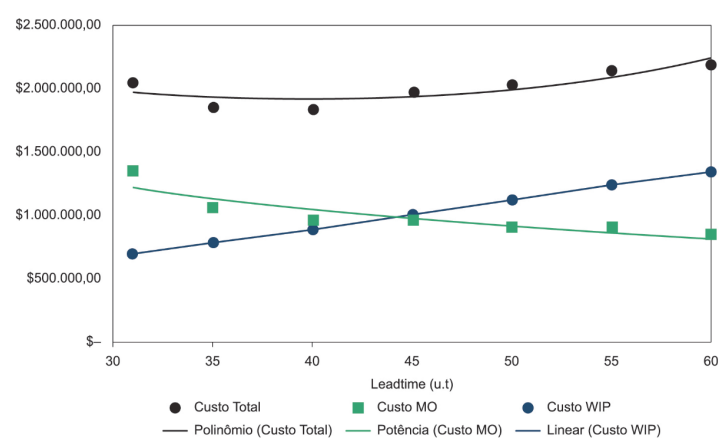

Figura 13. Variação do custo total em função do leadtime para o tempo de ciclo de 10 u.t.

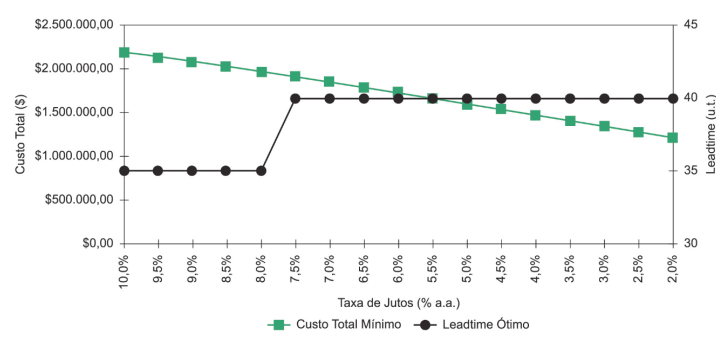

Figura 14. Variação do custo total em função do leadtime.

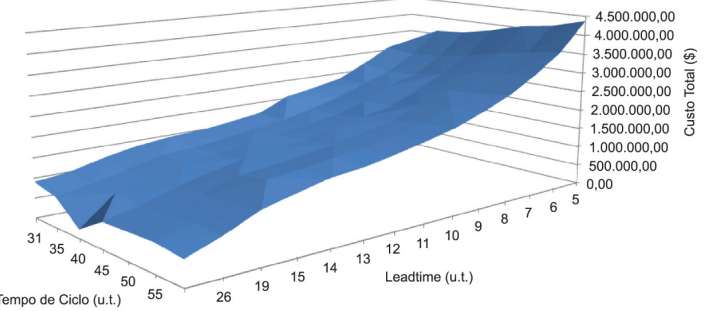

Figura 15. Variação do Custo Total Mínimo para o NS01.
Observe, comparando os resultados apresentados na Figura 8 e na Figura 13, que a taxa de ocupação da mão de obra aumenta conforme o leadtime aumenta. Entretanto, o custo total diminui somente até o leadtime 40 e começa a aumentar a partir deste, ilustrando um caso em que o aumento da taxa de ocupação da mão de obra aumenta os custos totais. Esse resultado contraria a prática da empresa, que tem o objetivo de sempre aumentar a taxa de ocupação da mão de obra (IUH - Índice de Utilização de Homem), supostamente para combater o desperdício da ociosidade de mão de obra, contudo, desconsidera o custo do WIP e o impacto dessa prática no custo total.

Como o custo do estoque em processo pode ser aproximado pelo custo de oportunidade de aplicar a quantidade de recursos financeiros imobilizada em estoques e aplicá-la no mercado financeiro a uma taxa de juros, pode ser feita uma análise de sensibilidade da taxa de juros com a finalidade de entender qual a variação no custo total e no leadtime da solução de custo total mínimo. Essa análise foi feita para o caso apresentado na Figura 13 e os resultados são apresentados na Figura 14. Conforme esperado, para taxas de juros maiores, a melhor solução é operar com leadtimes menores. Entretanto, para a faixa que vai de $7,5 \%$ a.a. até $2 \%$ a.a., utilizar o leadtime de 40 u.t. permanece como a melhor solução.

A Figura 15 apresenta a variação do custo total em função do leadtime e do tempo de ciclo para o NS01. $\mathrm{O}$ custo total diminui com o aumento do leadtime e do tempo de ciclo, o que seria esperado, pois essa é a combinação em que a quantidade de montadores e de estoque médio em processo diminui.

\section{Considerações finais}

Neste estudo abordou-se um problema de planejamento de linha de montagem estrutural aeronáutica de jatos por meio de modelos de programação de projetos. Diversos experimentos computacionais foram realizados utilizando-se casos realistas deste problema de montagem em uma empresa aeronáutica. Mostrou-se que o leadtime pode ter grande impacto na quantidade total de montadores, pois a sua escolha determina como será o nivelamento da mão de obra. Quanto maior o leadtime, maior a redução de mão obra devido ao melhor nivelamento. Entretanto, essa redução pode ter proporções bem diferentes para tempos de ciclo diferentes.

A análise baseada na aplicação de modelos de programação de projetos com recursos restritos e com únicos ou múltiplos modos de execução apresentou algumas vantagens. Uma delas foi que o modelo com múltiplos modos permitiu encontrar soluções para combinações de leadtimes e tempos de ciclo para as quais não há soluções factíveis com um único modo, comumente utilizado pela empresa. Além disso, quando ambos os modelos com único 
modo e múltiplos modos tinham soluções, em vários casos as programações com múltiplos modos eram melhores do que as programações com único modo, principalmente por utilizarem menos operadores.

Algumas comparações do custo de investir em capacidade (ou seja, ter leadtimes menores e contratar mais montadores) com o custo de aumentar os estoques em processo mostraram que é possível determinar pontos de equilíbrio ou compromisso entre esses custos. O custo de estoque em processo predomina sobre o custo de capacidade ociosa. Isso contrapõe o senso comum de que o aumento das taxas de ocupação dos recursos produtivos reduz os custos, pois desta maneira os custos fixos ficariam mais bem diluídos. Essa lógica não considera que o aumento das taxas de ocupação aumenta os leadtimes e os estoques que, no caso da indústria aeronáutica, são muito caros pelo alto valor agregado dos estoques em processo, que acabam aumentando os custos, ao invés de diminuí-los.

A variação na taxa de juros representando o custo de oportunidade influencia no ponto de equilíbrio ou trade-off entre capacidade e estoque. Porém, não altera as relações entre leadtimes e tempos de ciclo. Os modelos matemáticos utilizados neste estudo mostraram que é possível fazer análises mais aprofundadas, em nível tático, utilizando recursos computacionais aceitáveis para se gerar programas de produção ótimos ou quase ótimos.

Algumas perspectivas deste estudo para pesquisas futuras seriam: (i) aplicar a mesma abordagem de programação de projetos em outras montagens estruturais, incluindo linhas com diferentes tipos de aviões (linhas híbridas); (ii) avaliar o impacto do investimento em gabaritos e pesquisar e desenvolver outras formulações matemáticas de programação de projetos, por exemplo, representando o horizonte de planejamento em tempo contínuo em vez de discreto, com o objetivo de obter modelos mais representativos e computacionalmente mais fáceis de resolver. Outras perspectivas para pesquisa futura seriam considerar incertezas em alguns parâmetros dos modelos, como no leadtime, na duração de cada atividade e no tempo de ciclo, e avaliar os impactos na quantidade de mão de obra, estoque em processo e no trade-off entre o custo de investir em capacidade e o custo de estoque em processo. A avaliação desses critérios, juntamente com o critério de risco, poderia ser realizada utilizando técnicas de programação estocástica e de otimização robusta. Além dessas pesquisas, poder-se-ia também explorar o uso de heurísticas e meta-heurísticas para encontrar soluções factíveis nos casos em que os modelos matemáticos não encontraram, ou para permitir a obtenção de boas soluções em tempos computacionais menores do que os dos modelos.

\section{Agradecimentos}

Os autores agradecem aos revisores anônimos pelos úteis comentários e sugestões, e ao CNPq e à FAPESP pelo apoio a esta pesquisa.

\section{Referências}

Anzanello, M. J., \& Fogliato, F. S. (2007). Curvas de aprendizado: estado da arte e perspectivas de pesquisa. Gestão \& Produção, 14(1), 109-123. http://dx.doi. org/10.1590/S0104-530X2007000100010.

Arenales, M., Armentano, V., Morabito, R., \& Yanasse, H. (2007). Pesquisa operacional para cursos de engenharia. Rio de Janeiro: Campus.

Banks, J. (1998). Handbook of simulation: principles, methodology, advances, applications, and practice. New York: John Wiley \& Sons.

Brown, R., \& Caddick, J. (2002). The factory is virtual... The savings are real. In Symposium on "Reduction of Military Vehicle Acquisition Time and Cost through Advanced Modeling and Virtual Simulation (pp. 61-68). Paris. Recuperado em 16 de abril de 2013, de http:// www.dtic.mil/dtic/tr/fulltext/u2/p014151.pdf.

Brucker, P., Drexl, A., Möhring, R., Neumann, K., \& Pesch, E. (1999). Resource-constrained project scheduling: notation, classification, models, and methods. European Journal of Operational Research, 112(1), 3-41. http:// dx.doi.org/10.1016/S0377-2217(98)00204-5.

Davidz, H., \& Nightingale, D. (2002). AMRAAM case stude report - Lean Aerospace Initiative (LAI Report). Cambridge: Sociotechnical Systems Research Center. Recuperado em 16 de abril de 2013, de http://lean. mit.edu/downloads/doc_download/225-lean-effectson-aerospace-programs-leap-project-amraam-casestudy-report.

Ferdowsi, B., \& Haggerty, A. (2002). 737 Fuselage case study report - Lean Aerospace Initiative (LAI Report). Cambridge: Sociotechnical Systems Research Center. Recuperado em 16 de abril de 2013, de http://lean.mit. edu/downloads/doc_download/224-lean-effects-onaerospace-programs-leap-project-737-fuselage-casestudy-report.

Heike, G., Ramulu, M., Sorenson, E., Shanahan, P., \& Moinzadeh, K. (2001). Mixed model assembly alternatives for low-volume manufacturing: the case of aerospace industry. International Journal of Production Economics, 72(2), 103-120. http://dx.doi.org/10.1016/ S0925-5273(00)00089-X.

Herroelen, W., Reyck, B., \& Demeulemeester, E. (1998). Resource-constrained project scheduling: a survey of recent developments. Computers \& Operations Research, 25(4), 279-302. http://dx.doi.org/10.1016/ S0305-0548(97)00055-5.

Lennox, M. K. (2006). Implementing pull production within an aerospace assembly operation. (Dissertação de mestrado). Sloan School of Management, Massachusetts Institute of Technology, Massachusetts. 
Little, J. D. C. (1961). A proof for the queuing formula: $\mathrm{L}=\lambda \mathrm{W}$. Operations Research, 9(3), 383-387.

Morton, T. E., \& Pentico, D. W. (1993). Heuristic scheduling systems: with applications to production systems and project management. New York: John Wiley \& Sons.

Pinedo, M. L. (2008). Scheduling: theory, algorithms and systems (3 ed.). New-Jersey: Prentice- Hall.

Scott, H. A. (1994). Modeling aircraft assembly operations. In Proceedings of the Winter Simulation Conference (pp. 920-927), San Diego: Society for Computer Simulation International.

Silva, B. J. V. (2010). Programação de montagens em gabaritos com restrições de adjacência na indústria aeronáutica (Dissertação de mestrado). Departamento de engenharia de produção, Universidade Federal de São Carlos, São Carlos.

Silva, B. J. V., Morabito, R., \& Yanasse, H. H. (2011). Programação da produção de gabaritos de montagem com restrições de adjacência na indústria aeronáutica. Produção, 21(4), 696-707. http://dx.doi.org/10.1590/ S0103-65132011005000055.

Silva, B. J. V., Morabito, R., \& Yamashita, D. S. (2014a). Otimização na programação de montagens na indústria aeronáutica. Gestão \& Produção, 21(1), 33-44. http:// dx.doi.org/10.1590/S0104-530X2013005000002.

Silva, B. J. V., Morabito, R., Yamashita, D. S., \& Yanasse, H. H. (2014b). Production scheduling of assembly fixtures in aeronautical industry. Computers \& Industrial Engineering, 67, 195-203. http://dx.doi.org/10.1016/j. cie.2013.11.009.

Wolters, J. A., II. (2000). Optimizing the assembly sequence of an aerospace manufacturing process (Dissertação de mestrado). Sloan School of Management, Massachusets Institute of Technology, Massachusets.

Wright, T. P. (1936). Factors Affecting the Cost of Airplanes. Journal of the Aeronautical Sciences, 3(4), 122-128. http://dx.doi.org/10.2514/8.155.

Yamashita, D. S., \& Morabito, R. (2007a). Um algoritmo exato para o problema de programação de projetos com custo de disponibilidade de recursos e múltiplos modos. Pesquisa Operacional, 27(1), 27-49. http://dx.doi. org/10.1590/S0101-74382007000100003.

Yamashita, D. S., \& Morabito, R. (2007b). Um algoritmo branch-and-bound para o problema de programação de projetos com custo de disponibilidade de recursos e múltiplos modos. Gestão \& Produção, 14(13), 545-555.

Yamashita, D. S., \& Morabito, R. (2009). A note on time/ cost tradeoff curve generation for project scheduling with multi-mode resource availability costs. International Journal of Operational Research, 5(4), 429-444. http:// dx.doi.org/10.1504/IJOR.2009.025702.

Zhang, K., Cheng, H., \& Li, Y. (2008). Multi-objective harmonious colony-decision algorithm for more efficiently evaluating assembly sequences. Assembly Automation, 28(4), 348-355. http://dx.doi.org/10.1108/01445150810904503. 


\section{Errata/Erratum}

No artigo "Planejamento de uma montagem estrutural aeronáutica de jatos utilizando abordagens de programação de projetos com recursos restritos e múltiplos modos", com o DOI: http://dx.doi.org/10.1590/0104530X1739-14, publicado na Gestão \& Produção, Volume 22, número 2, página 254-266, na página 263, o autor solicitou a substituição das figuras, de modo que, onde se lia:

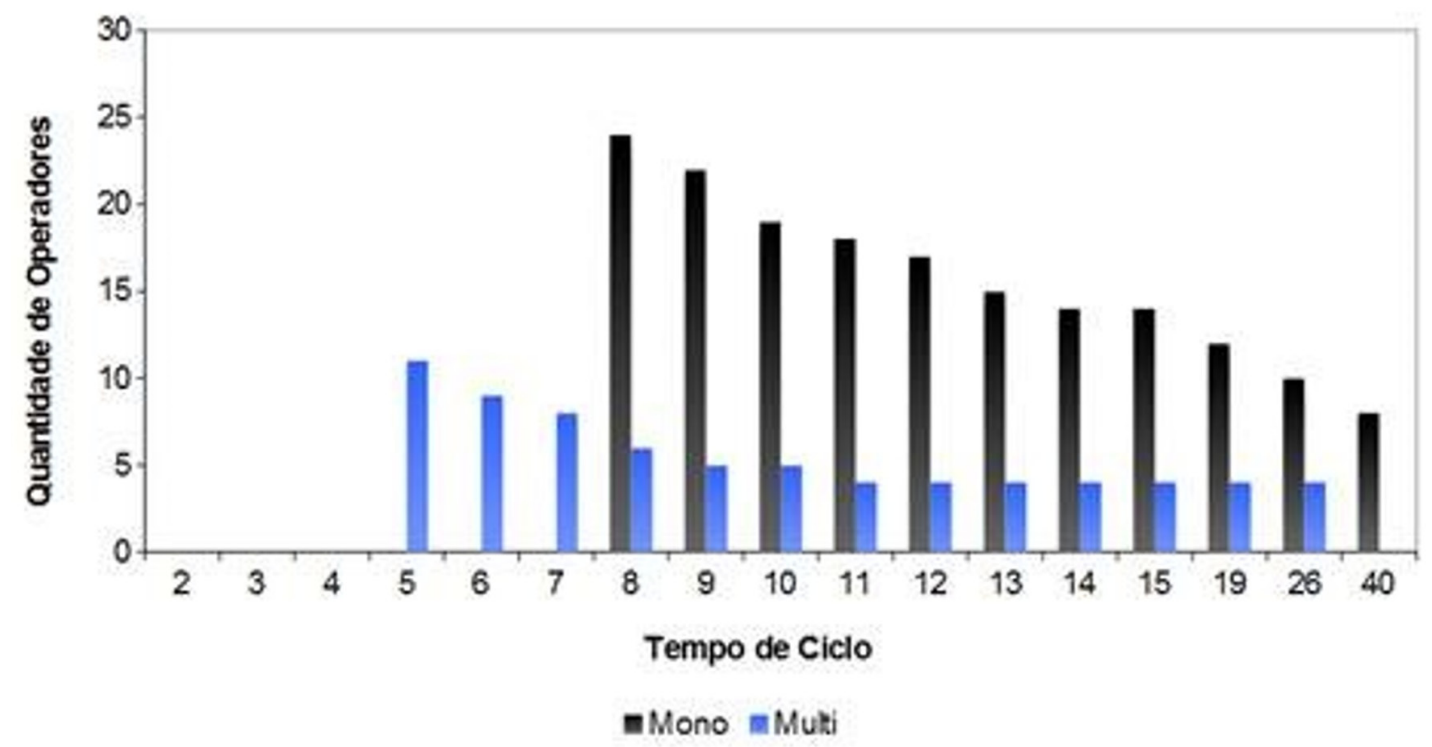

Figura 11: Original publicada no artigo, incorreta.

Leia-se:

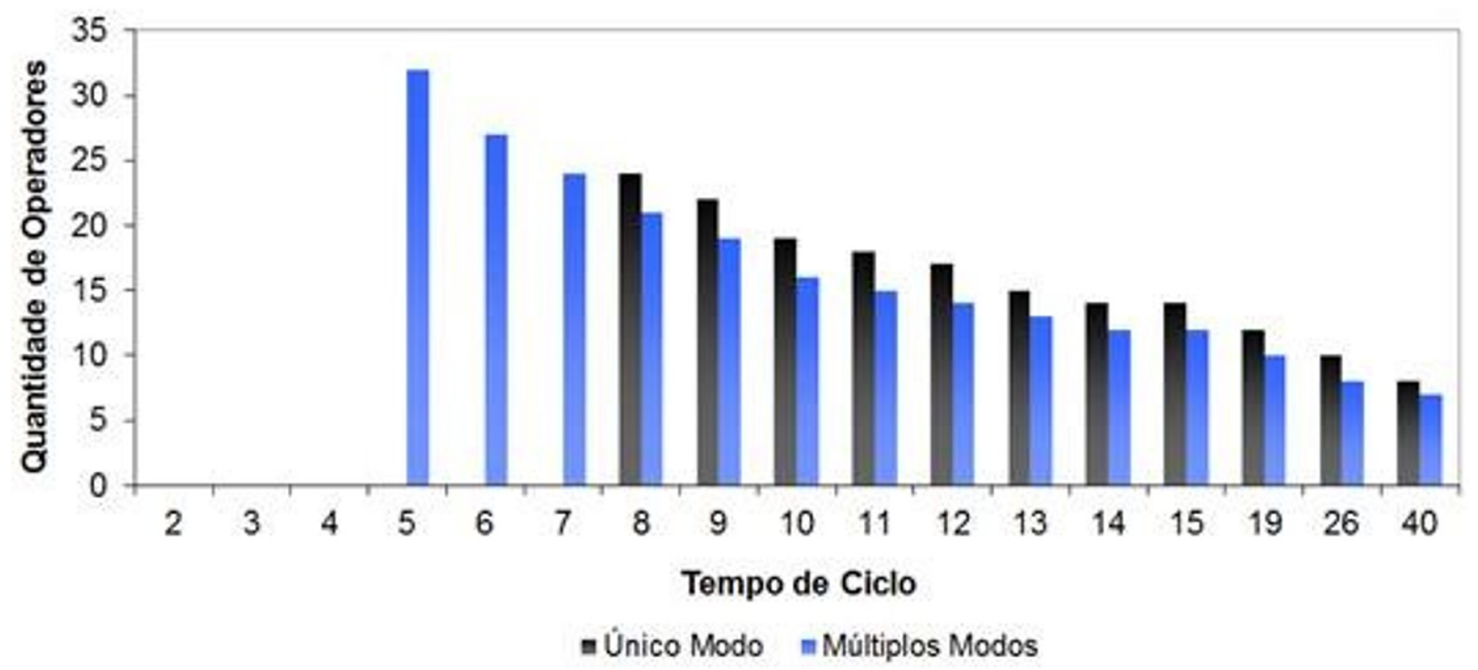

Figura 11: correta. 